\title{
A method to determine the mechanical properties of the retina based on an experiment in vivo
}

\author{
Xiuqing Qian ${ }^{\mathrm{a}, \mathrm{b}}$, Kunya Zhang ${ }^{\mathrm{a}, \mathrm{b}}$ and Zhicheng Liu ${ }^{\mathrm{a}, \mathrm{b}, *}$ \\ ${ }^{a}$ School of Biomedical Engineering, Capital Medical University, Beijing, 100069, China \\ ${ }^{\mathrm{b}}$ Beijing Key Laboratory of Fundamental Research on Biomechanics in Clinical Application, Capital \\ Medical University, Beijing, 100069, China
}

\begin{abstract}
A method is proposed to determine the mechanical properties of retina based on in vivo experiments and numerical simulations. First, saline water was injected into the anterior chamber of the right eye of a cat to cause acute high intraocular pressure. After the eye was scanned using optical coherence tomography under different acute high intraocular pressures, the images of the retina in vivo were obtained and the thickness of the retina was calculated. Then, the three-dimensional structure of the optic nerve head including the retina and the choroid were reconstructed using image processing technology. Three different material models for the retina and the choroid were taken and the finite element models of the optic nerve head were constructed. Finally, an inverse method was proposed to determine the parameters of a constitutive model of the retina and of the choroid simultaneously. The results showed that the deformation of the retina can be properly simulated taking into consideration the nonlinear elastic properties of the retina and of the choroid.
\end{abstract}

Keywords: Retina, choroid, mechanical properties, finite element method, optical coherence tomography

\section{Introduction}

Glaucoma is the second leading cause of blindness, after cataracts. The pathogenesis of glaucoma is not well understood, but mechanical compression or decreased blood flow in the optic nerve is considered the prime cause. When intraocular pressure increases, the optic nerve head is deformed because it is a weak spot in the posterior eye-wall. The lamina cribrosa, as the main structural element of the optic nerve head, bows outwards to an extent that depends on the magnitude of the pressure increase and on the resistance of the lamina to such distortion $[1,2]$. This deformation of the laminar structure depends on the elastic properties of the posterior ocular soft tissues [3]. The posterior segment wall of the eyeball has three layers: the sclera, the choroid, and the retina. The elastic properties of the sclera and choroid have attracted the attention of many researchers [4-8], including from our group [5], and the purpose of the present paper is to propose a method to determine the elastic properties of the retina.

\footnotetext{
* Address for correspondence: Zhicheng Liu, School of Biomedical Engineering, Capital Medical University, No. 10 Xitoutiao, You An Men, Beijing 100069, China. Tel.: +861083911559; Fax: +861083911560; E-mail: zcliu@ccmu.edu.cn.
} 
Biologic soft tissues are usually nonlinear, anisotropic, viscoelastic materials such as the retina and the choroid. The nonlinear properties of these materials can be described by J-shaped stress-strain curves, such as advanced prostheses and scaffolds for tissue engineering [9]. The viscoelasticity of the materials can be evaluated by the dynamic oscillatory test $[9,10]$. The storage modulus gives information about the material's ability to store elastic energy and the loss modulus is related to the viscosity or dissipation of energy. The retina is the inner tissue layer of eyeball, which is very fragile [11]. The choroid, lying between the retina and the sclera, is considerably stiffer than the retina and more resistant to mechanical deformation [12]. A classical approach to determine the mechanical properties is to perform a simple uniaxial test such as tensile or compression test, which involves significant simplifications and assumptions $[6,7,11,13-18]$. The mechanical properties of porcine retinal tissue have been studied using tensile tests and the results show that Young's modulus was a factor 10 higher in the choroid compared with the retina [18]. Other works show that mechanical characteristics of the porcine retina are anisotropic and inhomogeneous [13-15]. The results of Chen et al. show that the retina, choroid, and sclera behave nonlinearly, but only the retina exhibits surface anisotropy between the vertical and horizontal directions in body-temperature saline [14]. They also found that the size of the blood vessels affected the stiffness of the retina, and it is the only determining factor that governs the anisotropic and inhomogeneous characteristics of the retina [13].

Recently, the material properties of ocular posterior soft tissues were studied using new technology. Jones et al. obtained the Young's modulus of bovine retina by fitting the experiment data from the in vitro tensile test of bovine retina to a mathematical model [19]. Shahbazi, et al. proposed a noninvasive and safe method to estimate the elastic modulus of the retina-choroid complex in normal and age-related macular degeneration human subjects using temporal sequences of ultrasound images [20]. Ugarte and his colleague developed a new experimental method to obtain the stress-strain relation of the human Bruch's membrane-choroid complex [8]. The pressure was measured using a modified Ussing chamber and the deformation was examined by optical coherence tomography (OCT). A method based on the wrinkling periodicity of epiretinal membranes and contraction-induced choroidal folds to study the elastic properties of the retina and of the choroid showed that the Young's modulus of the choroid is larger than the retina's [12]. Scanning force microscopy was used to measure the local mechanical properties of the inner surface of the guinea pig retina [21]. By comparing the spreading behavior of a liquid on the retina with that of the same liquid on each of two viscoelastic materials, Grant et al. estimated the elastic moduli of the retina [22].

Most of these authors studied the mechanical properties of the retina and of the choroid at room temperature. The results of Chen and colleagues show that the elastic modulus of ocular posterior soft tissues are different in different environments, such as room temperature air, room-temperature saline, and body temperature saline [14]. They also examined the mechanical characteristics of pig retina at $26.1^{\circ} \mathrm{C}$ and $7.8^{\circ} \mathrm{C}$, and found that the retina is significantly stiffer at a low temperature than at body temperature [18]. These results illustrate the importance of testing conditions. Therefore, it is important to develop a method based on in vivo experiments to determine the material properties of the retina.

A more recent approach is to combine numerical and experimental techniques to determine the material properties of biological soft tissues indirectly [23]. To find out the material parameters of the skin in vivo, a method that consists in combining experimental indentation results with a numerical finite elements model was proposed by Delalleau and his colleagues [24]. Similarly, a new technique was developed to determine the properties of porcine corneas in vivo, using a combination of nanoindentation experimentation, an anisotropic and orthotropic Generalized Maxwell model, and an inverse finite element method [25]. The virtual field method, for instance, has been developed for 
extracting constitutive parameters based on full-field measurements using the optical non-contact techniques [26]. Nguyen and Boyce proposed an inverse method to determine the anisotropic properties of bovine cornea by combining an in vitro inflation experiment and finite element method [27]. They measured the three-dimensional surface geometry and displacement field of the cornea using a digital image correlation system, which included two cameras mounted stereoscopically above the specimen.

Precise biomechanical characterization of soft tissues has recently attracted much attention in medical image analysis and visualization. To get to the undeformed state, a custom inverse model was developed using a finite element method analysis package and clinical topography measurements in the patient eyes [28]. A three-dimensional model of the cornea was constructed using tomographic data from an anterior segment imaging system. Based on these works, an inverse system of estimating longitudinal, time-dependent changes in the corneal elastic modulus after crosslinking was developed [29]. Our group proposed a method to determine the material properties of the iris using the multiisland genetic algorithm coupled with the finite element method based on the ultrasonic image in vivo [30].

With the development of OCT [31,32], a new technology, known as enhanced depth-imaging OCT, has been developed to reliably capture the image of the full thickness of the choroid in various retinal diseases [33-35]. Image quality of the choroid in vivo could be improved by displacing the instrument to get images in deeper layers. An "Enhanced Choroidal Mode" of the Topcon's OCT visualizes further internal structures, allowing significantly superior visualization of the retinal pigment epithelium and choroidal area [36]. Our group has obtained the effect of elevated intraocular pressure on the thickness changes of the laminar and prelaminar tissue of cats using optical coherence tomography [37]. It is thus possible to develop an inverse method to determine the material properties of the retina and of the choroid in vivo. In this study, based on high-resolution images of the optic nerve head using the "Enhanced Choroidal Mode" of the Topcon's OCT, a method is proposed to determine the material properties of retina using the finite element method and the optimization algorithm.

\section{Method}

\subsection{Experiment in vivo}

The sample is from the right eye of a cat from the Animal Center of Capital Medical University with the level of specific pathogen free. In this research, the animals were treated and managed to comply with the ARVO statement for the use of animals in ophthalmic and vision research. The intraocular pressure (IOP) of the cat was measured using pressure transducer (SPR-320; Millar Instruments Inc., Houston, USA) after anesthesia. The value of IOP was obtained using the Powerlab data acquisition system and Chart software; it was $15 \mathrm{~mm} \mathrm{Hg}$. The Powerlab data acquisition system (PowerLab 8/30) with eight input channels is suitable for a wide range of research applications. The cat was mounted in a stereotaxic head holder and its anterior chamber was perfused with physiological saline. To avoid movement of the eye, a topical anesthetic and a mydriatic mixture were dropped on the surface of the cornea. A pressure transducer was placed in the saline injection port and the real-time intraocular pressure in the perfused eye of the cat was measured using the Powerlab data acquisition system and Chart software. The pressure was gradually increased to a peak $(120 \mathrm{~mm} \mathrm{Hg})$ using an experiment system shown in Figure 1 from normal IOP. The IOP was monitored in real time during the infusion. 


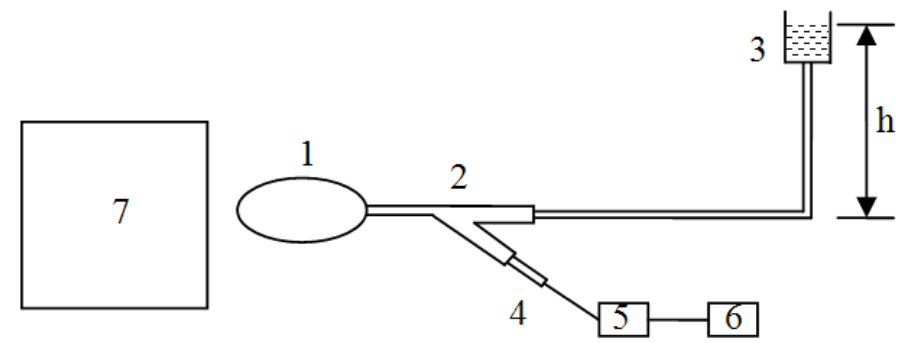

Fig. 1. Sketch of the experimental apparatus. 1. Anterior chamber of eyeball; 2. Detaining needle 3. Medicine flask; 4. Pressure transducer; 5. Powerlab data acquisition system; 6. Compuer (Chart software); 7. OCT.

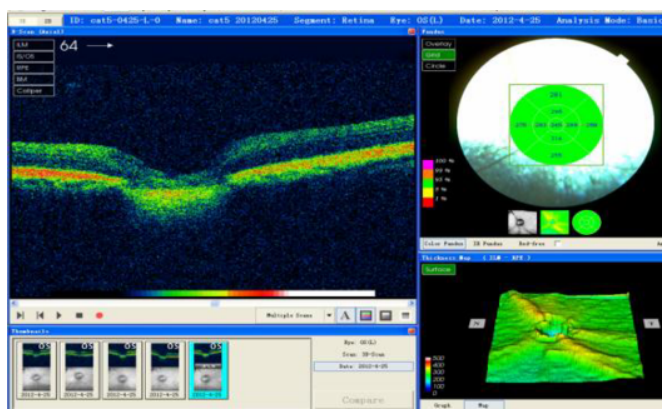

Fig. 2. The spectral domain (SD) optical coherence tomography (OCT) images of the optic nerve head: The upper left image is one of the tomogram of OCT. There are 128 images. The right lower image is the thickness contour of the retina.

The infusion was briefly paused (for approximately 15-30 s) at the IOP of 40,60,80,100, and 120 $\mathrm{mm} \mathrm{Hg}$ to stabilize IOP and scan by OCT. For each eye, the total measurement was completed within 30 min for six replicates.

In this study, the images were obtained at the different IOP values using the "Enhanced Choroidal Mode" of the Topcon's OCT. The images were acquired by a continuous parallel scan function focused on the rectangular region of the optic nerve head. The serial images were then reconstructed during the optic nerve head surface spacing of $6.0 \times 6.0 \times 2.3 \mathrm{~mm}$ (horizontal $\times$ vertical $\times$ axial). Finally, three-dimensional volumetric matrixes with $512 \times 128 \times 650$ pixels were obtained, as shown in Figure 2. The scan was performed six times (six different IOP values).

The algorithm to calculate the thickness of the retina was coded using MATLAB (The Mathworks, Inc., Natick, MA, USA). First, the image segmentation was done using the optimal threshold

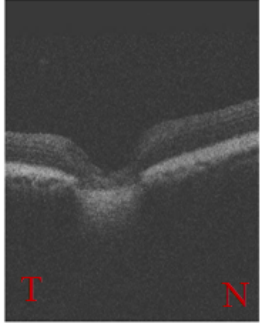

(a)

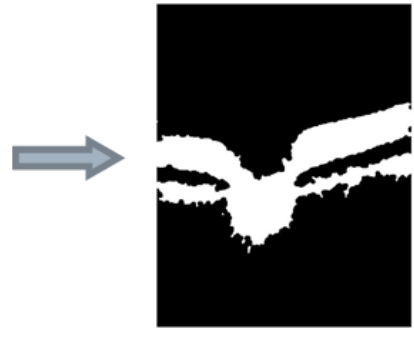

(b)

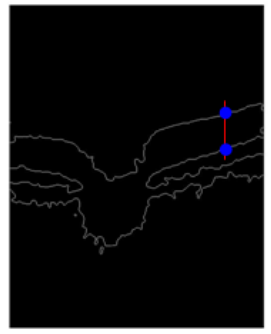

(c)

Fig. 3. Image processing (a) Orign image. T: temporal side; N: nasal side. (b) Image filling (c) Edge extracting. The thickness of the retina is calculated according to the distance of two point in (c). 
segmentation algorithm program after choosing the maximum and minimum value of the threshold. Then, the edge was extracted using the Canny edge detector after the image was filled. Finally, the thickness was calculated according to the upper and lower boundaries, as shown in Figure 3.

\subsection{Finite element model}

According to the serial images obtained by OCT for the normal IOP, a three-dimensional model of the retina and of the choroid was built using MIMICS (version 14.12; Materialise, Leuven, Belgium). Then, a three-dimensional model of the optic nerve head containing the retina and the choroid was assembled, as shown in Figure 4.

Next, the model was input to finite element software ABAQUS (version 6.12; Simulia). The finite element model included 271012 elements and 58690 nodes. According to the research of Jones and colleagues [19], the retina can be modeled as incompressible material. Therefore, a four-node linear tetrahedron, hybrid element was applied, which can describe the quasi-incompressible mechanical response of the material. The interactive boundary condition between the retina and the choroid was set as boned. Considering the effect of the sclera, the spring constrain at the bottom of the choroid was added.

Although the relationship between the stress and strain of retina is nonlinear according to some previous studies, it is difficult to find the suitable constitutive model to appropriately characterize these properties [13]. Thus, three elasticity models were chosen to study the mechanical characteristics of the retina. One is the linear elastic model, another is the Ogden model, and the third is the neoHookean material model.

In the Ogden model, the strain energy function is written as [38]

$$
U=\sum_{i=1}^{N} \frac{2 \mu_{i}}{\alpha_{i}^{2}}\left(\bar{\lambda}_{1}^{\alpha_{i}}+\bar{\lambda}_{2}^{\alpha_{i}}+\bar{\lambda}_{3}^{\alpha_{i}}-3\right)+\sum_{i=1}^{N} \frac{1}{D_{i}}\left(J_{e l}-1\right)^{2 i}
$$

where $U$ is the strain energy density per unit volume in the undeformed configuration, $\mu_{i}, \alpha_{i}$ and $D_{i}$ are material parameters, $J$ is the volume ratio, $\bar{\lambda}_{i}$ is the deviatoric principal stretch which is related to the principal stretch $\lambda_{i}$ by $\bar{\lambda}_{i}=J^{-\frac{1}{3}} \lambda_{i}$.

The neo-Hookean material model can be expressed as:
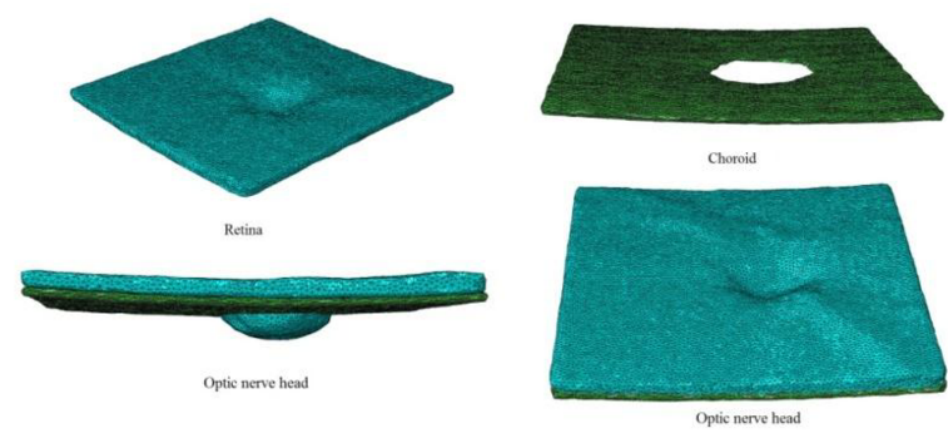

Fig. 4. Three-dimensional reconstruction of the optic nerve head: The upper left figure is the retina, and the upper right figure is the choroid. The two lower figures are assembled of the retina and of the choroid. 


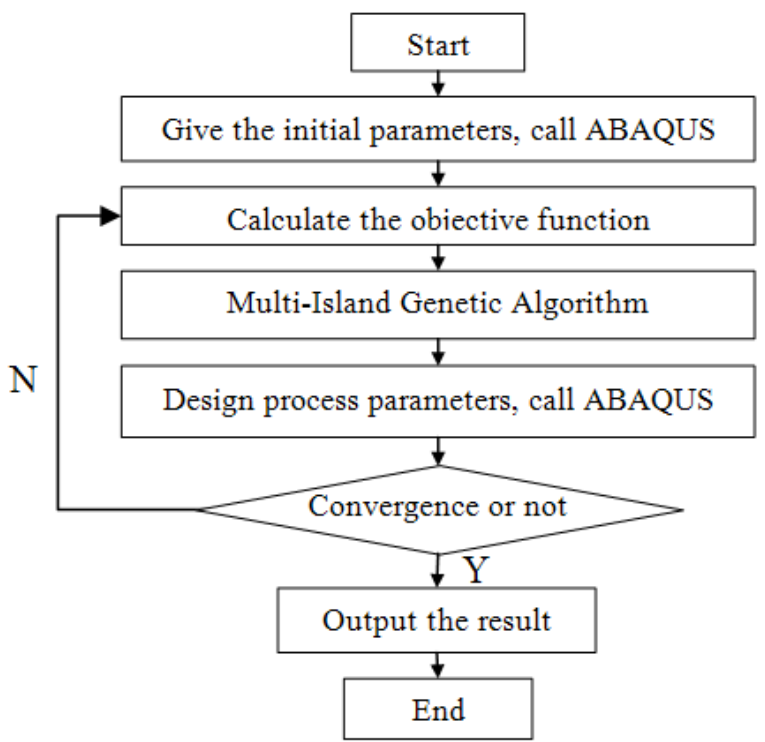

Fig. 5. The flowchart of optimization.

$$
\Phi=C_{10}\left(\bar{I}_{1}-3\right)+\frac{1}{D}(J-1)^{2}
$$

\subsection{Optimization process}

The optimization process to identify material properties is implemented by means of iSIGHT (version 5.7; Simulia), integrated with ABAQUS. The multi-island genetic algorithm is used to determine the material properties of the retina and of the choroid in iSIGHT. As a kind of improved genetic algorithm, the multi-island genetic algorithm divides the population in one generation into several sub-populations called 'islands' and performs traditional genetic operations on each island separately [39]. Figure 5 shows the flowchart of optimization. The objective function used in the optimization algorithm is

$$
J(\vec{x})=\frac{1}{2} \sum_{\mathrm{i}}\left(\boldsymbol{u}_{\mathrm{r}}^{\mathrm{sim}}\left(p_{\mathrm{i}}\right)-\mathcal{u}_{\mathrm{r}}^{\exp }\left(p_{\mathrm{i}}\right)\right)^{2}
$$

Where the $\mathcal{u}_{\mathrm{r}}^{\text {sim }}$ is the deformation of the retina calculated using the finite element method and the $u_{\mathrm{r}}^{\exp }$ is the deformation of the retina measured using OCT. We define the error as the difference between $\mathcal{u}_{\mathrm{r}}^{\text {sim }}$ and $\mathcal{u}_{\mathrm{r}}^{\text {exp }}$.

In this paper, the settings of the multi-island genetic algorithm are as follows: the number of islands is 2 , the number of generations is 10 , the size of populations is 25 , the crossover rate is 1 , the mutation rate is 0.01 , the migration rate is 0.01 , and the migration interval is 5 .

\section{Results and discussion}




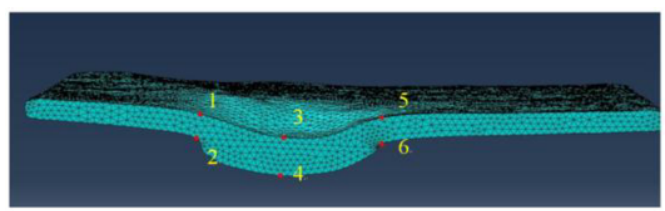

Fig. 6. Three typical locations to calculate the thickness of the retina: Point 4 is the lowest point of ONH; Point 2 is the position with the most acute change in curvature at the left of the image; Point 6 is the position with the most acute change in curvature at the right of the image; Points 1,3, and 5 are above Points 2, 4, and 6.

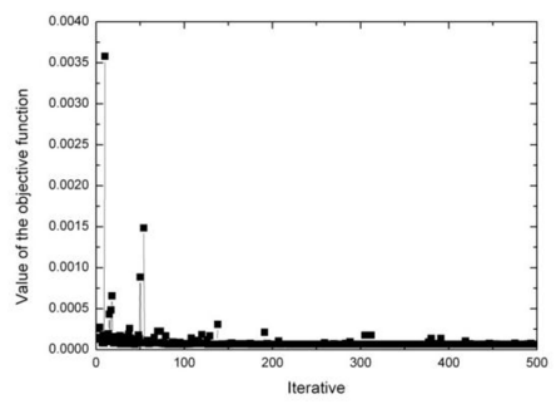

Fig. 7. Convergence after 500 times iterative calculation.

The deformation at three typical locations was chosen to identify the material properties of the retina and of the choroid, as shown in Figure 6. First, we searched the image, which included the lowest point, such as point 4 in Figure 6. Generally, it is located in the middle of the optic nerve head $(\mathrm{ONH})$. Then, the point at the same position on the upper surface of the ONH was searched, such as point 3 in Figure 6. The distance between point 3 and point 4 can denote the thickness of the middle position of the ONH. Similarly, the thickness at the left side of the optic disk was defined as the distance between point 1 and point 2 . The thickness at the right side of the optic disk was defined as the distance between point 5 and point 6 . Computation of retina thickness was achieved using the method in 2.1

Considering the material properties of the soft tissue, the values of the parameter Di in Eq. (1) and D in Eq. (2) are taken as 0 for all the materials in this paper [40]. The parameter identification process is shown in Figure 5. Figure 7 shows that the objective convergence is good after 500 times iterative calculation when the materials' models was taken as a second-order Ogden function. The materials' parameters obtained are presented in Tables 1-3.

Table 1

Materials parameters obtained using the Ogden material model

\begin{tabular}{l|l|l|l|l}
\hline Materials Name & $\mu_{1}(\mathrm{MPa})$ & $\alpha_{1}$ & $\mu_{2}(\mathrm{MPa})$ & $\alpha_{2}$ \\
\hline Retina & 0.0811 & 0.00227 & 0.0652 & 0.00421 \\
\hline Choroid & 143.9 & 52530.7 & 179.7 & 76383.6 \\
\hline
\end{tabular}

Table 2

Material parameters obtained using the linear elastic material model

\begin{tabular}{l|l|l}
\hline Materials Name & $E(\mathrm{MPa})$ & $\mu$ \\
\hline Retina & 0.431 & 0.416 \\
\hline Choroid & 4.62 & 0.46 \\
\hline
\end{tabular}


Table 3

Material parameters obtained using the neo-Hookean material model

\begin{tabular}{l|l}
\hline Materials Name & $\mathrm{C}_{10}(\mathrm{MPa})$ \\
\hline Retina & 0.0759 \\
\hline Choroid & 29.0 \\
\hline
\end{tabular}

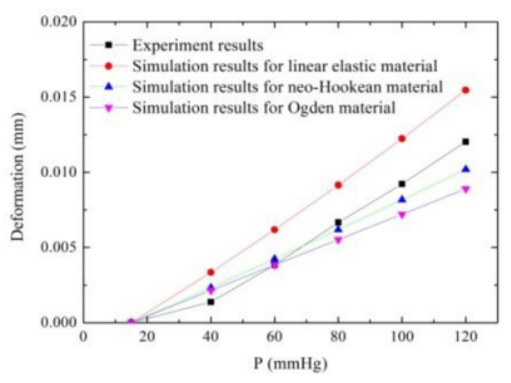

(a)

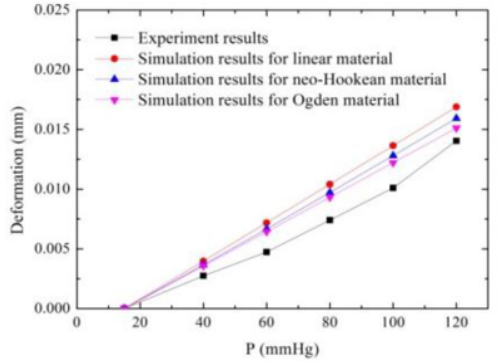

(b)

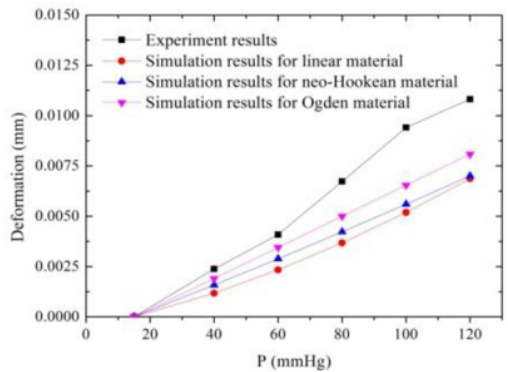

(c)

Fig. 8. Errors between the experiment results and the simulation results: (a) At the left side of the optic disk; (b) middle position of $\mathrm{ONH}$; and (c) at the right side of the optic disk.

To verify the reasonability of the identified result, the variation of the thickness of the three positions on $\mathrm{ONH}$ was calculated and is shown in Figure 6. The errors between the experiment data and the simulation results are shown in Figure 8. The results show that the material properties of retina and choroid processed through the Ogden model can simulate the deformation of the retina the best. The error is larger if we take the linear elasticity as the material model. Therefore, the deformation of the retina can be properly simulated considering the nonlinear elastic properties of the retina and of the choroid.

Although the mechanical properties of the retina are believed to be nonlinear by some researchers [13-16, 18], a common understanding for the right constitutive model of the retina has not yet been reached. Mechanical properties of the retina are simplified as linear elasticity in most studies. The values of the elastic modulus of the retina are in a large range, from 0.3 to $125 \mathrm{KPa}[13,14,16,18,20$, 21], which may be due to different measurement methods, different species, and different assay conditions. For example, the results of the elastic modulus of porcine retina obtained using scanning force microscopy in vivo is smaller than if a simple tension test is used $[4,13,18,21]$. The results of the elastic modulus of porcine retina are also different for different temperatures in simple tension tests [18]. Moreover, the geometry of the biological samples is important in mechanical testing. For example, the retinal thicknesses were not uniform even for the same specimen [11]. The shape of the specimen is also a factor affecting experiment results. For example, $5 \mathrm{~mm} / 13 \mathrm{~mm}$ was taken in [11] 
for bovine eyes and $1.5 \mathrm{~mm} / 10 \mathrm{~mm}$ was taken in [14].

The elastic modulus of the choroid is $4.62 \mathrm{MPa}$ and the elastic modulus of the retina is $0.431 \mathrm{MPa}$ for the linear elasticity hypothesis in our paper, and the difference of the elastic modulus between the retina and the choroid was significant, which is consistent with Chen's results [14]. The elastic modulus of the choroid is close to the results of Chen's experimental result of the porcine choroid in vitro [14]. The elastic modulus of the retina is different from the tension test in vitro, and we think the reason may be that the thickness of the retinal specimen may have been too small and there were larger errors in the tension test. We obtained the experiment data in vivo, avoiding errors involved in the process of specimen preparation, and the results obtained is reasonable and in line with other studies $[14,16,18]$.

\section{Conclusions}

An inverse method was proposed to determine the material properties of the retina through combined computational and experimental techniques. The material parameters of retina and choroid were obtained by minimizing the error between the experimental data and the simulation results. The experiment results were obtained by OCT and the simulation results were obtained by a finite element method. It is found that the Ogden function can be used to describe the materials properties of the retina.

\section{Acknowledgment}

This work was financially supported by National Natural Science Foundation of China Nos. 11102123 and 31070840, and by the Natural Science Foundation of Beijing Nos. 7142024 and 3122010 .

\section{References}

[1] H.A. Quigley and E.M. Addicks, Regional differences in the stucture of the laminal cribeosa and their relation to glaucomatous optic nerve damage, Archives of Ophthalmology 99 (1981), 137-143.

[2] C. Balaratnasingam, W.H. Morgan, L. Bass, G. Matich, S.J. Cringle and D.Y. Yu, Axonal transport and cytoskeletal changes in the laminar regions after elevated intraocular pressure, Investigative Ophthalmology \& Visual Science 48 (2007), 3632-3644.

[3] I. A. Sigal and C. R. Ethier, Biomechanics of the optic nerve head, Experimental Eye Research 89 (2009), $799-807$.

[4] B. Geraghty, S.W. Jones, P. Rama, R. Akhtar and A. Elsheikh, Age-related variations in the biomechanical properties of human sclera, Journal of the Mechanical Behavior of Biomedical Materials 16 (2012), 181-191.

[5] M.M. Wang, F.J. Zhang, X.Q. Qian and X. Zhao, Regional biomechanical properties of human sclera after cross-linking by UVA/Riboflavin, Journal of Refractive Surgery 28 (2012), 723-728.

[6] W.P. Graebel and G.W.H.M. van Alphen, The elasticity of sclera and choroid of the human eye, and its implications on scleral rigidity and accommodation, Journal of Biomechanical Engineering 99 (1977), 203-208.

[7] T.R. Friberg and J.W. Lace, A comparison of the elastic properties of human choroid and sclera, Experimental Eye Research 47 (1988), 429-436.

[8] M. Ugarte, A.A. Hussain and J. Marshall, An experimental study of the elastic properties of the human Bruchs membrane-choroid complex, British Journal of Ophthalmology 90 (2006), 621-626.

[9] A. Gloria, R. De Santis, L. Ambrosio, F. Causa and K.E. Tanner, A multi-component fiber-reinforced PHEMA-based hydrogel/HAPEX ${ }^{\mathrm{TM}}$ device for customized intervertebral disc prosthesis, Journal of Biomaterials Applications 25 (2011), 795-810. 
[10] A. Borzacchiello, A. Gloria and L. Mayol, Natural/synthetic porous scaffold designs and properties for fibrocartilaginous tissue engineering, Journal of Bioactive and Compatible Polymers 26 (2011), 437-451.

[11] W. Wu, W.H. III Peters and M.E. Hammer, Basic mechanical properties of retina in simple elongation, Journal of Biomechanical Engineering 109 (1987), 65-67.

[12] L.V. Del Priore, Stiffness of retinal and choroidal tissue a surface wrinkling analysis of epiretinal membranes and choroidal folds, American Journal of Ophthalmology 142 (2006), 435-440.

[13] K. Chen and J.D. Weiland, Anisotropic and inhomogeneous mechanical characteristics of the retina, Journal of Biomechanics 43 (2010), 1417-1421.

[14] K. Chen, A.P. Rowley and J.D. Weiland, Elastic properties of porcine ocular posterior soft tissues, Journal of Biomedical Materials Research Part A 93 (2010), 634-645.

[15] K. Chen, A.P. Rowley, J.D. Weiland and M.S. Humayun, Elastic properties of human posterior eye, Journal of Biomedical Materials Research Part A 102 (2014), 2001-2007.

[16] G. Wollensak and E. Spoerl, Biomechanical characteristics of retina, Retina-The Journal of Retinal and Vitreous Diseases 24 (2004), 967-970.

[17] G. Wollensak, E. Spoerl, G. Grosse and C. Wirbelauer, Biomechanical significance of the human internal limiting lamina, Retina-The Journal of Retinal and Vitreous Diseases 26 (2006), 965-968.

[18] K. Chen and J.D. Weiland, Mechanical characteristics of the porcine retina in low temperatures, Retina-The Journal of Retinal and Vitreous Diseases 32 (2012), 844-847.

[19] I.L. Jones, M. Warner and J.D. Stevens, Mathematical modelling of the elastic properties of retina: A determination of young's modulus, Eye 6 (1992), 556-559.

[20] S. Shahbazi, M. Mokhtari-Dizaji and M.R. Mansori, Noninvasive estimation of the ocular elastic modulus for agerelated macular degeneration in the human eye using sequential ultrasound imaging, Ultrasonics 52 (2012), 208-214.

[21] K. Franze, M. Francke, K. Günter, A.F. Christ, N. Körber, A. Reichenbach and J. Guck, Spatial mapping of the mechanical properties of the living retina using scanning force microscopy, Soft Matter 7 (2011), 3147-3154.

[22] C.A. Grant, P.C. Twigg, M.D. Savage, W.H. Woon, M. Wilson and D. Greig, Estimating the mechanical properties of retinal tissue using contact angle measurements of a spreading droplet, Langmuir 29 (2013), 5080-5084.

[23] F. Lei and A.Z. Szeri, Inverse analysis of constitutive models: Biological soft tissues, Journal of Biomechanics 40 (2007), 936-940.

[24] A. Delalleau, G. Josse, J.M. Lagarde, H. Zahouani and J.M. Bergheau, Characterization of the mechanical properties of skin by inverse analysis combined with the indentation test, Journal of Biomechanics 39 (2006), 1603-1610.

[25] M.H. Abyaneha, R.D. Wildmanb, I.A. Ashcroftb and P.D. Ruiza, A hybrid approach to determining cornea mechanical properties in vivo using a combination of nano- indentation and inverse finite element analysis, Journal of the Mechanical Behavior of Biomedical Materials 27 (2013), 239-248.

[26] M. Gre'diac, F. Pierron, S. Avril and E.Toussaint, The virtual field method for extracting constitutive parameters from full-field measurements: A review, Strain 42 (2006), 233-253.

[27] T.D. Nguyen and B. Boyce, An inverse finite element method for determining the anisotropic properties of the cornea, Biomechanics and Modeling in Mechanobiology 10 (2011), 323-337.

[28] A.S. Roy and W.J. Dupps Jr, Patient-specific modeling of corneal refractive surgery outcomes and inverse estimation of elastic property changes, Journal of Biomechanical Engineering 133 (2011), 011002.

[29] A.S. Roy, K.M. Rocha, J.B. Randleman, R.D. Stulting and W.J. Dupps Jr, Inverse computational analysis of in vivo corneal elastic modulus change after collagen crosslinking for keratoconus, Experimental Eye Research 113 (2013), 92104.

[30] K.Y. Zhang, X.Q. Qian, X. Mei and Z.C. Liu, An inverse method to determine the mechanical properties of the iris in vivo, BioMedical Engineering Online 13 (2014), doi: 10.1186/1475-925X-13-66.

[31] N.G. Strouthidis, B. Fortune, H. Yang, I.A. Sigal and C.F. Burgoyne, Longitudinal change detected by spectral domain optical coherence tomography in the optic nerve head and peripapillary retina in experimental glaucoma, Investigative Ophthalmology \& Visual Science 52 (2011), 1206-1219.

[32] I. Maruko, T. Iida, Y. Sugano, M. Saito and T. Sekiryu, Subfoveal retinal and choroidal thickness after verteporfin photodynamic therapy for polypoidal choroidal vasculopathy, American Journal of Ophthalmology 151 (2011), 594603.

[33] H.L. Yang, J.J. Qi, C. Hardin, S.K. Gardiner, N.G. Strouthidis, B. Fortune and C.F. Burgoyne, Spectral-domain optical coherence tomography enhanced depth imaging of the normal and glaucomatous nonhuman primate optic nerve head, Investigative Ophthalmology \& Visual Science 53 (2012), 394-405.

[34] H.Y.L. Park, S.H. Jeon and C.K. Park, Enhanced depth imaging detects lamina cribrosa thickness differences in normal tension glaucoma and primary open-angle glaucoma, Ophthalmology 119 (2012), 10-20.

[35] S.C. Park, C.G.V. De Moraes, C.C. Teng, C. Tello, J.M. Liebmann and R. Ritch, Enhanced depth imaging optical 
coherence tomography of deep optic nerve complex structures in glaucoma, Ophthalmology 119 (2012), 3-9.

[36] T. Yamashita, T. Yamashita, M. Shirasawa, N, Arimura, H, Terasaki and T, Sakamoto, Repeatability and reproducibility of subfoveal choroidal thickness in normal eyes of Japanese using different SD-OCT devices, Investigative Ophthalmology \& Visual Science 53 (2012), 1102-1107.

[37] Q.Y. Zhao, X.Q. Qian, L. Li, W.J. Sun, S. Huang and Z.C. Liu, Effect of elevated intraocular pressure on the thickness changes of cat laminar and prelaminar tissue using optical coherence tomography, Bio-Medical Materials and Engineering 24 (2014), 2349-2360.

[38] R.W. Ogden, Large deformation isotropic elasticity on the correlation of theory and experiment for incompressible rubberlike solids, Proceedings of the Royal Society A-Mathematical Physical and Engineering Sciences 326 (1972), 565-584.

[39] M. Miki, T. Hiroyasu, M. Kaneko and K. Hatanaka, A parallel genetic algorithm with distributed environment scheme, IEEE International Conference on Systems, Man, and Cybernetics 1 (1999), 695-700.

[40] ABAQUS, Analysis User's Manual Version 6.12, Dassault Systèmes Simulia Corp., Providence, Rhode Island, USA, 2012. 